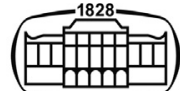

AKADÉMIAI KIADÓ

Acta Veterinaria

Hungarica

$68(2020) 3,251-256$

DOl:

10.1556/004.2020.00047

(c) 2020 Akadémiai Kiadó, Budapest

\section{CASE REPORT}

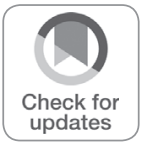

*Corresponding author.

E-mail: kangbt@chungbuk.ac.kr Tel.:

+8243261 3744; fax: +82 43267 2595

\title{
Combination therapy with azathioprine, cyclosporine and ketoconazole in a dog with concurrent pemphigus foliaceus and hyperadrenocorticism - Case report
}

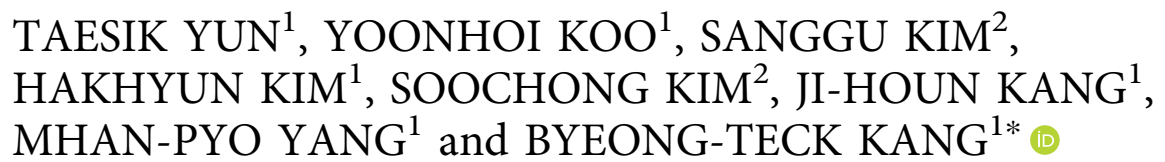

${ }^{1}$ Laboratory of Veterinary Internal Medicine, College of Veterinary Medicine, Chungbuk National University, Cheongju, Chungbuk 28644, South Korea

${ }^{2}$ Department of Veterinary Medicine, College of Veterinary Medicine, Chungbuk National University, Cheongju, Chungbuk 28644, South Korea

Received: September 19, 2019 • Accepted: September 23, 2020

Published online: November 21, 2020

\begin{abstract}
A 10-year-old, spayed female Shih Tzu dog presented with a history of progressive erythema and multiple crusts developing 85 days previously. The dog had been diagnosed with hyperadrenocorticism (HAC) 55 days prior to presentation and was treated with oral trilostane $(2.86 \mathrm{mg} / \mathrm{kg}$, once daily) that was discontinued due to a poor response. In addition to generalised alopecia, erythematous plaques and crusts were noted on the trunk, head and footpads. Lesional impression smears revealed numerous acantholytic cells and non-degenerated neutrophils. Histopathological findings demonstrated subcorneal pustules with acantholytic cells and intact neutrophils. On the basis of these findings, we diagnosed pemphigus foliaceus (PF) with concurrent HAC. We wished to avoid glucocorticoids and, therefore, prescribed oral, once-daily azathioprine $(2 \mathrm{mg} / \mathrm{kg})$, modified cyclosporine $(7 \mathrm{mg} / \mathrm{kg})$ and ketoconazole $(5 \mathrm{mg} / \mathrm{kg})$. By day 71 post-treatment, the erythematous crusts had almost disappeared and the alopecia had improved considerably. However, by the subsequent follow-up examination on day 99, the clinical signs had reappeared due to the tapering of cyclosporine. To the best of our knowledge, this is the first case report describing concurrent PF and HAC in a dog. Combination therapy with azathioprine, modified cyclosporine and ketoconazole was effective, and should be considered for dogs diagnosed with concurrent autoimmune diseases and HAC.
\end{abstract}

\section{KEYWORDS}

azathioprine, cyclosporine, dog, hyperadrenocorticism, ketoconazole, pemphigus foliaceus

\section{INTRODUCTION}

Pemphigus foliaceus (PF) is an autoimmune skin disorder characterised by the production of autoantibodies targeting intercellular desmosomes on keratinocytes (Hnilica and Patterson, 2016). PF is usually treated by systemic immunosuppressive therapy using glucocorticoids alone or in combination with additional immunosuppressive agents (Mueller et al., 2006).

Hyperadrenocorticism (HAC) is an endocrinopathy showing the typical clinical signs caused by hypercortisolism (chronic overproduction of cortisol by the adrenal cortices) (Alenza and Melián, 2017). Common clinical signs include polyphagia, polyuria, polydipsia, muscle weakness, panting and abdominal enlargement (Behrend, 2015). Cutaneous changes, such as hyperpigmentation, non-pruritic alopecia and secondary pyoderma, may also present in HAC (Zur and White, 2011). HAC is usually treated by inhibition of cortisol production 
through lysis of the adrenal cortex or pharmaceutical inhibition of cortisol biosynthesis (Alenza and Melián, 2017).

Many cases of iatrogenic HAC have been reported in dogs undergoing glucocorticoid treatment for autoimmune disease. However, the co-occurrence of autoimmune disease in dogs with non-iatrogenic HAC is very rare. In this paper we report an interesting case of $\mathrm{PF}$ with concurrent naturally occurring HAC and assess the efficacy of an alternative to glucocorticoid therapy for this combined disorder.

\section{CASE REPORT}

A 10-year-old, 3.5-kg, spayed female Shih Tzu dog with a history of HAC was referred to us with progressive skin lesions. The dog had shown only multifocal dermatologic signs, including pustules, crusts and ulceration, 85 days prior to presentation. At the first animal hospital, the dog was diagnosed with superficial pyoderma presumptively resulting from endocrinopathy and treated with cephalexin and amoxicillin-potassium clavulanate. Despite receiving treatment for bacterial infection, the dermatologic signs deteriorated and the dog was referred to a second animal hospital for identification of the endocrinopathy-related dermatosis. At the second animal hospital, 55 days before presentation to our institution, an adrenocorticotropic hormone (ACTH) stimulation test was performed, showing a baseline serum cortisol concentration of $>276 \mathrm{nmol} / \mathrm{L}$ (reference range: 55.2-165.6 nmol/L), and a corresponding value of $>828$ nmol/L after ACTH stimulation (reference range: 165.6$496.8 \mathrm{nmol} / \mathrm{L}$ ). Ultrasonographic evaluation of the adrenal glands showed left and right adrenal gland widths of 6 and $5.2 \mathrm{~mm}$, respectively. On dermatologic examination, an impression smear showed numerous acantholytic cells, nondegenerated neutrophils and some phagocytic neutrophils including cocci. Fungal culture using a dermatophyte test medium and Sabouraud dextrose agar was performed, and the results were negative. A complete blood count and biochemical profile showed moderate leucocytosis $(29.38 \times$ $10^{9} / \mathrm{L}$; reference range: $5.05-16.76 \times 10^{9} / \mathrm{L}$ ), with mild neutrophilia $\left(14.38 \times 10^{9} / \mathrm{L}\right.$; reference range: $2.95-11.64 \times$ $\left.10^{9} / \mathrm{L}\right)$, moderate monocytosis $\left(3.75 \times 10^{9} / \mathrm{L}\right.$; reference range: $\left.0.16-1.12 \times 10^{9} / \mathrm{L}\right)$ and moderate lymphocytosis $\left(10.99 \times 10^{9} / \mathrm{L}\right.$; reference range: $\left.1.05-5.10 \times 10^{9} / \mathrm{L}\right)$, as well as mildly increased alkaline phosphatase activity (311 IU/L; reference range: 29-97 IU/L). On the basis of these results, the dog was diagnosed with superficial pyoderma due to HAC and treated using oral trilostane $(2.86 \mathrm{mg} / \mathrm{kg}$, once daily), marbofloxacin, cephalexin and metronidazole. Itraconazole was also prescribed, although fungal cultures were negative.

Despite receiving treatment for 55 days at the second animal hospital, the clinical signs deteriorated. For this reason, the dog was referred to our institution. We repeated the ACTH stimulation test on admission to identify the state of HAC. Pre- and post-ACTH serum cortisol concentrations were 240 and $270 \mathrm{nmol} / \mathrm{L}$, respectively. Physical examination on admission revealed erythematous, crusting dermatitis of the head, face, trunk, forelimbs and hindlimbs, as well as generalised alopecia (Fig. 1A and B). Hyperkeratosis of all footpads and bilateral corneal ulcers were present. On dermatologic examination, an impression smear of

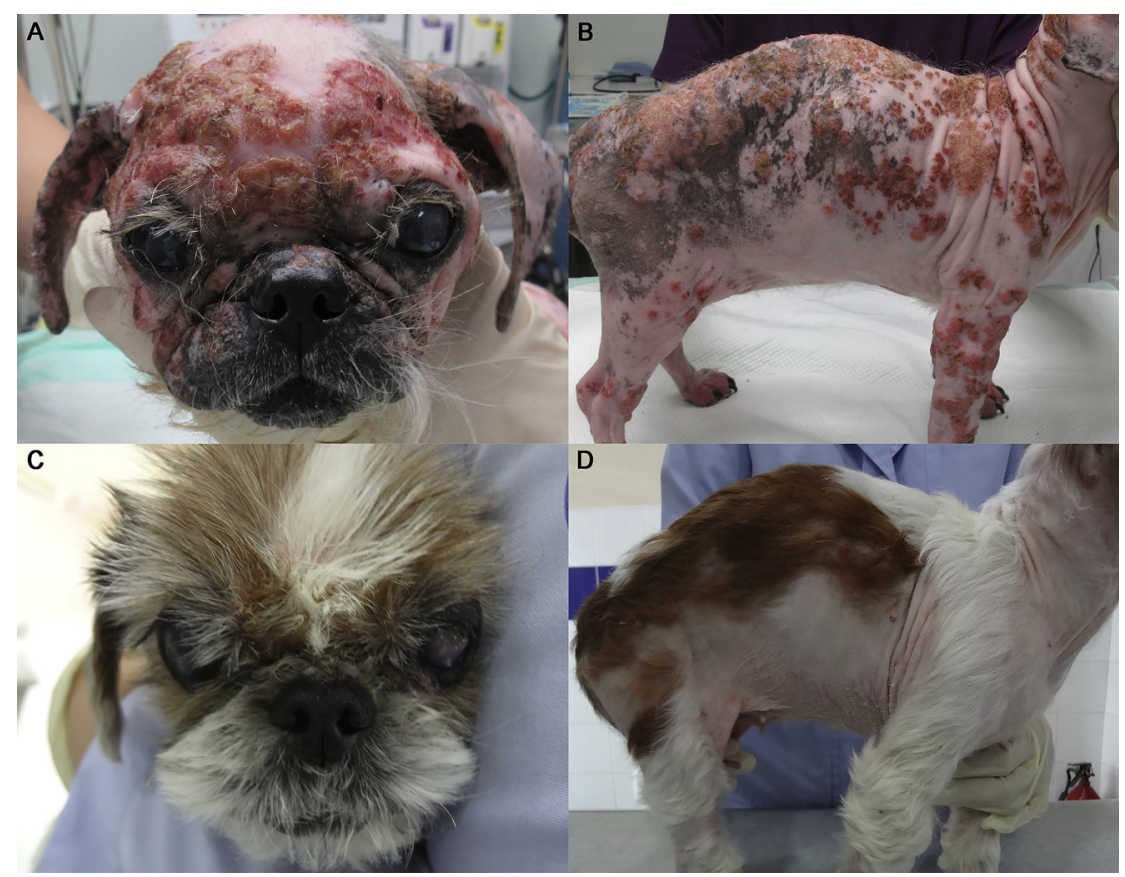

Fig. 1. Typical skin lesions of pemphigus foliaceus in a dog with hyperadrenocorticism, at initial presentation and after immunosuppressive treatment using oral once-daily azathioprine, modified cyclosporine and ketoconazole. (A, B) Severe generalised alopecia from hyperadrenocorticism and multiple erythematous crusts on the head, trunk, forelimbs and hindlimbs before treatment. (C, D) Almost complete resolution of lesions at 71 days after immunosuppressive treatment 


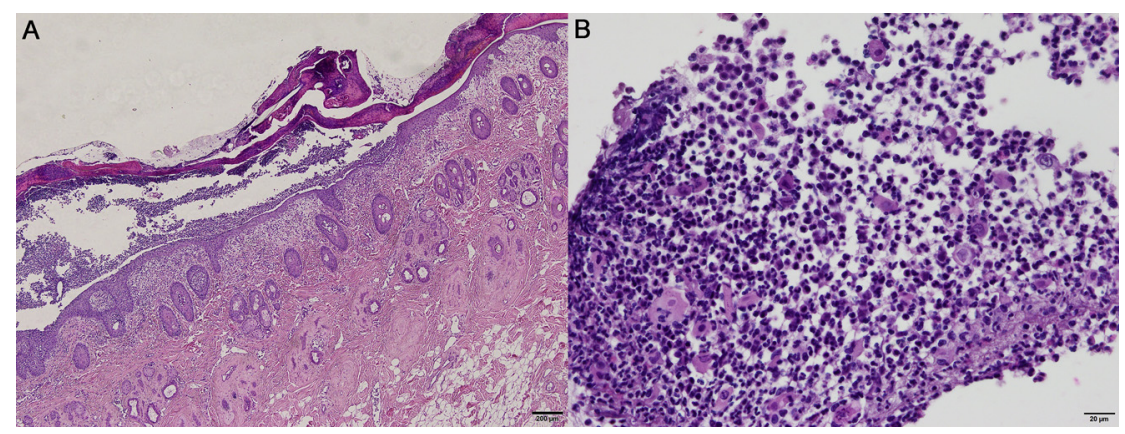

Fig. 2. Histopathological findings of pemphigus foliaceus in a dog. (A) Head. Separation of the stratum corneum and subcorneal pustule, with numerous non-degenerated neutrophils. Haematoxylin and eosin (HE). (B) Head. A number of free-floating acantholytic cells with eosinophilic cytoplasm and numerous non-degenerated neutrophils (HE)

remarkable lesions revealed numerous acantholytic cells and non-degenerated neutrophils. Bacterial culture was performed, and Sphingomonas paucimobilis was grown. Other tests such as Wood's lamp examination, a trichogram, hair fungal culture on dermatophyte test medium and Sabouraud dextrose agar, and skin scrapings were negative for dermatophytosis and ectoparasites. A complete blood count and biochemical profile revealed severe monocytosis $(10.45 \times$ $10^{9} / \mathrm{L}$; reference range: $\left.0.16-1.12 \times 10^{9} / \mathrm{L}\right)$, severe lymphocytosis $\left(14.98 \times 10^{9} / \mathrm{L}\right.$; reference range: $\left.1.05-5.10 \times 10^{9} / \mathrm{L}\right)$, severe neutrophilia $\left(38.17 \times 10^{9} / \mathrm{L}\right.$; reference range: $2.95-$ $11.64 \times 10^{9} / \mathrm{L}$ ), moderate anaemia (packed cell volume: $21.1 \%$; reference range: $37.3 \%-61.7 \%)$, moderate hypoalbuminaemia (17 g/L; reference range: 26-33 g/L) and moderately increased alkaline phosphatase activity (534 IU/ L; reference range: 29-97 IU/L). We obtained punch skin biopsy samples from the head and left lateral elbow. The dog was initially treated with trimethoprim-sulphamethoxazole (30 mg/kg, orally, twice daily; Septrin tab., Samil Pharm., Seoul, South Korea), terbinafine $(30 \mathrm{mg} / \mathrm{kg}$, orally, twice daily; Lamisil Tab., Novartis, Barcelona, Spain), ivermectin (0.3 mg/kg, orally, once daily; Ivomec, Merial, Lyon, France) and bathing with an antibacterial shampoo (MALASEB-F, Dermcare-Vet Pty Ltd., Australia) for infectious skin diseases before the biopsy results came back 11 days later. Despite this treatment, the dermatologic signs did not improve. Histopathological examination revealed prominent subcorneal pustules containing well-preserved neutrophils and a number of acantholytic cells (Fig. 2A). Detachment of acantholytic keratinocytes from the stratum spinosum was evident at the base of the pustule. Superficial lymphocytic infiltrates with occasional eosinophils were observed in the dermis. Acantholytic cells were characterised by round to polyhedral shape with abundant hypereosinophilic cytoplasm and a viable nucleus (Fig. 2B). The dog also showed orthokeratotic hyperkeratosis and follicular keratosis suggestive of HAC. On the basis of these combined findings and the history of poor response to treatment for infectious skin disease, the dog was diagnosed as showing PF with concurrent HAC, and immunosuppressive therapy was indicated. Because the dog had HAC and bilateral corneal ulcers, to avoid the side effects of glucocorticoids, the dog was treated initially once daily with oral azathioprine $(2 \mathrm{mg} / \mathrm{kg}$; Immuthera Tab., Celltrion Pharm Inc., Cheongju, South Korea), modified cyclosporine (7 mg/kg; Cipol-N, Chong Kun Dang Pharm. Corp., Seoul, South Korea), ketoconazole (5 mg/kg; Ketoconazole Tablets, Eaglevet, Seoul, South Korea) and amoxicillin-potassium clavulanate $(25 \mathrm{mg} / \mathrm{kg}$; Clavet-50, Cipla, Mumbai, India), while discontinuing trimethoprim-sulphamethoxazole. Ketoconazole was used to both elevate serum cyclosporine levels and help manage the HAC. Trilostane was discontinued due to a poor response and side effects such as lethargy and hyporexia. Follow-up examination on day 11 after initiation of immunosuppressive therapy revealed disappearance of more than $50 \%$ of the erythematous crusts, and slow improvement of the generalised alopecia. An impression smear of the lesion also showed numerous acantholytic cells and intact neutrophils. Blood analysis showed similar results as the first examination; severe neutrophilia $\left(30.64 \times 10^{9} / \mathrm{L}\right.$; reference range: $2.95-11.64 \times 10^{9} / \mathrm{L}$ ), moderate anaemia (packed cell volume: $23.8 \%$; reference range: 37.3-61.7\%), moderate hypoalbuminaemia (17 g/L; reference range: $26-33 \mathrm{~g} / \mathrm{L}$ ), and mildly increased alkaline phosphatase activity (318 IU/L; reference range: 29-97 IU/L). The dog was maintained on the above doses of azathioprine, modified cyclosporine, ketoconazole and amoxicillin-potassium clavulanate. A third ACTH stimulation test on day 37 after immunosuppressive therapy showed pre- and post-ACTH serum cortisol concentrations of $<30$ and $89 \mathrm{nmol} / \mathrm{L}$, respectively. Progressive improvements of the skin lesions until day 47 after immunosuppressive therapy permitted tapering of the oral modified cyclosporine and ketoconazole doses to 7 and 5 $\mathrm{mg} / \mathrm{kg}$ every other day, respectively, while maintaining the doses of azathioprine and amoxicillin-potassium clavulanate. By day 71 post-treatment, more than $90 \%$ of the erythematous crusts had disappeared, and the alopecia had improved considerably without adverse events (Fig. 1C and D). We decided to maintain the dose of medications until the next follow-up.

By the time of the next follow-up examination on day 99, however, new erythematous crusts had appeared on the trunk, and hyperkeratosis of the footpads had worsened. An impression smear of the lesions showed numerous 
acantholytic cells and intact neutrophils. Laboratory examinations showed severe neutrophilia $\left(32.86 \times 10^{9} / \mathrm{L}\right.$; reference range: $2.95-11.64 \times 10^{9} / \mathrm{L}$ ), moderate anaemia (packed cell volume 25.0\%; reference range: 37.3-61.7\%), mild hypoalbuminaemia ( $21 \mathrm{~g} / \mathrm{L}$; reference range: $26-33 \mathrm{~g} / \mathrm{L})$ and moderately increased alkaline phosphatase activity (986 IU/ L; reference range: 29-97 IU/L). This dermatologic deterioration prompted us to discontinue the combination therapy of azathioprine, modified cyclosporine and ketoconazole with amoxicillin-potassium clavulanate, and to change the immunosuppressive treatment to mycophenolate mofetil monotherapy $(20 \mathrm{mg} / \mathrm{kg}$, orally, twice daily; CellCept, Roche, Basel, Switzerland) with trimethoprim-sulphamethoxazole (30 mg/kg, orally, twice daily; Septrin tab., Samil Pharm., Seoul, South Korea). Partial response was maintained during a further 35 days of this treatment, but the patient was then lost to follow-up.

\section{DISCUSSION}

To the best of our knowledge, this is the first report of concurrent PF and non-iatrogenic HAC in a dog. While cases of autoimmune disease, such as Graves' disease, Hashimoto thyroiditis, psoriasis, systemic lupus erythematosus, rheumatoid arthritis, myasthenia gravis and Horton disease, accompanied by Cushing's syndrome (CS) have been reported in humans (Petramala et al., 2018), such reports are rare in veterinary medicine. Concurrence of autoimmune disease and CS may be obscure and not be recognised until the remission of CS, because endogenous glucocorticoids are favourable for suppressing activated immune function. It is known that autoimmune diseases may be newly diagnosed after remission of hypercortisolism (Petramala et al., 2018) and that the clinical signs of autoimmune diseases generally improve as a result of excess endogenous glucocorticoids in human patients with CS (Arima et al., 1998; Senécal et al., 1994). In contrast, the present case showed no improvement in the clinical signs of PF despite the hypercortisolism from HAC. Therefore, we considered it likely that this dog was refractory to glucocorticoids.

The most effective systemic immunosuppressive therapies for PF generally use glucocorticoids alone or in combination with additional immunosuppressive agents (Mueller et al., 2006). In the current case, because the dog presented with HAC and bilateral corneal ulcers and was thought to be refractory to glucocorticoids, the application of glucocorticoids was contraindicated. As an alternative, we attempted combination therapy with other immunosuppressive agents. Azathioprine is the first choice of glucocorticoid-sparing agents (Mueller et al., 2006). However, although azathioprine has been used as a drug to achieve final control of PF in some cases, it had been applied initially in combination with other immunosuppressive agents whose doses were gradually tapered and ceased (Mueller et al., 2006). Cyclosporine had also been used as a single immunosuppressive agent in PF, but it showed limited efficacy in one study (Rosenkrantz et al., 1989). In comparison with monotherapy of each drug, a combined regimen of azathioprine and cyclosporine was reported to be effective in human glucocorticoid-resistant ulcerative colitis (Campbell and Ghosh, 2003) and canine refractory PF (Rosenkrantz and Aniya, 2007). For these reasons, we decided to use combination therapy of azathioprine and modified cyclosporine instead of monotherapy and used ketoconazole to both elevate serum cyclosporine levels and also help manage the HAC.

Although PF usually occurs spontaneously, it may also be related to drug administration. Previously, drug-related PF was observed in dogs receiving trimethoprim-sulphonamide, cephalexin and oxacillin (White et al., 2002). Specifically, the skin eruptions always occur after the onset of suspected antibiotic treatment. In the present case, we considered the possibility of a pemphigus-like drug reaction to cephalexin, trimethoprim-sulphamethoxazole and trilostane. However, because the same dermatological signs were first noted before the application of cephalexin, trimethoprim-sulphamethoxazole and trilostane, the condition that we report is likely to be a spontaneous PF rather than a drug-induced eruption.

$\mathrm{PF}$ is characterised by presence of autoantibodies targeting the desmosomal proteins of keratinocytes, resulting in separation of keratinocytes. In human patients with PF, the majority of circulating immunoglobulin $G$ autoantibodies target conformational epitopes on the desmosomal cadherin desmoglein-1 (Koulu et al., 1984). However, indirect immunofluorescence of canine PF sera showed that antidesmoglein-1 autoantibodies were rare in dogs (Olivry et al., 2006). Therefore, desmoglein-1 is a minor autoantigen in canine PF compared to humans. Desmocollin-1 is also a calcium-dependent cadherin for adhesion between keratinocytes in the epidermis. The epidermis in desmocollin-1deficient mice showed weakened adhesion, including epidermal fragility and acantholysis, similar to human PF (Chidgey et al., 2001). A recent study reported that canine $\mathrm{PF}$ was characterised by the production of immunoglobulin $\mathrm{G}$ antibodies against desmocollin-1 (Bizikova et al., 2012). In this study, the proportion of anti-desmocollin-1 immunoglobulin $\mathrm{G}$ antibodies in canine PF was significantly higher than that in healthy dogs. Therefore, the identification of anti-desmocollin-1 autoantibodies, not anti-desmogelin-1 autoantibodies, can be a specific marker for the diagnosis of canine PF.

The most important diseases to be differentiated from PF are staphylococcal pyoderma, demodicosis and dermatophytosis. At the time of writing this report, there were no serum samples to identify anti-desmocollin-1 autoantibodies to specifically diagnose PF. However, since the typical clinical signs were evident, a diagnosis of PF could be readily made. Before presentation to our institution, long-term (85day) treatments for bacterial and fungal infection were performed in the first and second animal hospitals. Moreover, we repeated treatment against bacterial, fungal and ectoparasitic diseases for 11 days. Despite receiving this 
long-term treatment for infectious diseases, the dog showed poor response and deterioration of dermatologic signs. Furthermore, because bacterial pyoderma, which is a representative pustular disease, does not cause crusting of the footpads or involve the ears, infectious agents were thought to not contribute to this case. Some retrospective studies have also diagnosed PF on the basis of histopathological findings (pustules with marked acantholytic cells) with history and physical examination features suggestive of $\mathrm{PF}$ and ruling out infectious skin diseases (Gomez et al., 2004; Mueller et al., 2006). In the present case, the dog was diagnosed with PF on the basis of all these facts.

Although the relapse rate of canine PF has not been reported, that of human PF was reported to be $67 \%$ (Ujiie et al., 2019). In human PF, a relapse would occur as the dose of prednisolone is tapered to approximately $0.1 \mathrm{mg} / \mathrm{kg}$ (Ujiie et al., 2019). If a relapse or poor response to therapy is identified, the possibility of a secondary infection or demodicosis should be considered. Because we not only examined for secondary infection and demodicosis but also prescribed antibiotics, the cause of clinical recurrence in our case was suspected to be the tapering of the cyclosporine dose. However, further studies are needed to identify the efficacy and relapse rate of this combination therapy in the future.

In the present case, abnormal results in the complete blood count and the biochemical profile, such as neutrophilic leucocytosis, hypoalbuminaemia and increase in alkaline phosphatase activity, were identified and persisted during the treatment period. The relationship between PF and the findings of blood analysis has been rarely investigated. Vaughan et al. (2010) reported the results of a complete blood count and biochemical profile performed in 28 dogs before the initial treatment. Major findings were neutrophilic leucocytosis (64\%), hypoalbuminaemia (25\%), increased serum alkaline phosphatase activity (21\%), increased serum alanine transaminase activity (4\%), hypercholesterolaemia (4\%) and azotaemia (4\%). Although the abnormal results in this case may have been affected by HAC, they were most likely due to the combination effect of $\mathrm{PF}$ and HAC.

The low-dose dexamethasone suppression test is known to be much more sensitive in diagnosing HAC than the ACTH stimulation test (Behrend et al., 2013), but its use for diagnosis in the present case was precluded by the absence of the low-dose dexamethasone suppression test at the previous animal hospital and our desire to use comparable diagnostic methods.

This is the first reported case of concomitant PF with HAC in veterinary medicine. Although complete remission was not shown, our findings suggest that combination therapy with azathioprine, modified cyclosporine and ketoconazole may be considered an alternative to glucocorticoids for refractory patients, or for cases in which glucocorticoid therapy is limited for other reasons. Further studies will be required to investigate the pathogenesis of concurrent $\mathrm{PF}$ and HAC in canine patients.

\section{ACKNOWLEDGEMENTS}

The authors thank Seo-Jin Park for valuable assistance and care of the study dog. This work was carried out with the support of Basic Science Research Program through the National Research Foundation of Korea (NRF) funded by the Ministry of Education (2017R1D1A3B03028863).

\section{REFERENCES}

Alenza, D. P. and Melián, C. (2017): Hyperadrenocorticism in dogs. In: Ettinger, S. J., Feldman, E. C. and Côté, E. (eds) Textbook of Veterinary Internal Medicine, Vol. 2. 8th ed. Elsevier, Missouri, USA. pp. 1795-1811.

Arima, K., Higuchi, M., Yoshizawa, S., Horiuchi, T., Nagasawa, K., Nakashima, H., Taniguchi, M. and Niho, Y. (1998): Improvement of systemic lupus erythematosus activity by the association of delayed onset Cushing's syndrome. J. Rheumatol. 25, 2456-2458.

Behrend, E. N. (2015): Canine hyperadrenocorticism. In: Feldman, E. C., Nelson, R. W. and Reusch, C. E. (eds) Canine and Feline Endocrinology. 4th ed. Elsevier, Missouri, USA. pp. 377-451.

Behrend, E. N., Kooistra, H. S. and Nelson, R. (2013): Diagnosis of spontaneous canine hyperadrenocorticism: 2012 ACVIM consensus statement (small animal). J. Vet. Intern. Med. 27, 1292-1304.

Bizikova, P., Dean, G. A., Hashimoto, T. and Olivry, T. (2012): Cloning and establishment of canine desmocollin-1 as a major autoantigen in canine pemphigus foliaceus. Vet. Immunol. Immunopathol. 149, 197-207.

Campbell, S. and Ghosh, S. (2003): Combination immunomodulatory therapy with cyclosporine and azathioprine in corticosteroid-resistant severe ulcerative colitis: the Edinburgh experience of outcome. Dig. Liver Dis. 35, 546-551.

Chidgey, M., Brakebusch, C., Gustafsson, E., Cruchley, A., Hail, C., Kirk, S., Merritt, A., North, A., Tselepis, C., Hewitt, J., Byrne, C., Fassler, R. and Garrod, D. (2001): Mice lacking desmocollin 1 show epidermal fragility accompanied by barrier defects and abnormal differentiation. J. Cell Biol. 155, 821-832.

Gomez, S. M., Morris, D. O., Rosenbaum, M. R. and Goldschmidt, M. H. (2004): Outcome and complications associated with treatment of pemphigus foliaceus in dogs: 43 cases (19942000). J. Am. Vet. Med. Assoc. 224, 1312-1316.

Hnilica, K. A. and Patterson, A. P. (2016): Small Animal Dermatology: A Color Atlas and Therapeutic Guide. 4th ed. Elsevier, Missouri, USA. pp. 245-301.

Koulu, L., Kusumi, A., Steinberg, M. S., Klaus-Kovtun, V. and Stanley, J. R. (1984): Human autoantibodies against a desmosomal core protein in pemphigus foliaceus. J. Exp. Med. 160, 1509-1518.

Mueller, R. S., Krebs, I. and Power, H. T. (2006): Pemphigus foliaceus in 91 dogs. J. Am. Anim. Hosp. Assoc. 42, 189-196.

Olivry, T., LaVoy, A., Dunston, S. M., Brown, R. S., Lennon, E. M., Warren, S. J., Prisayanh, P., Müller, E. J., Suter, M. M. and 
Dean, G. A. (2006): Desmoglein-1 is a minor autoantigen in dogs with pemphigus foliaceus. Vet. Immunol. Immunopathol. 110, 245-255.

Petramala, L., Olmati, F. and Conforti, M. G. (2018): Autoimmune diseases in patients with Cushing's syndrome after resolution of hypercortisolism: case reports and literature review. Int. J. Endocrinol. 2018, 1464967.

Rosenkrantz, W. S. and Aniya J. S. (2007): Cyclosporine, ketoconazole and azathioprine combination therapy in three cases of refractory canine pemphigus foliaceus. North Am. Vet. Dermatol. Forum Proceed. 220, 2007.

Rosenkrantz, W. S., Griffin, C. E. and Barr, R. J. (1989): Clinical evaluation of cyclosporine in animal models with cutaneous immune-mediated disease and epitheliotropic lymphoma. J. Am. Anim. Hosp. Assoc. 25, 377-384.
Senécal, J. L., Uthman, I. and Beauregard, H. (1994): Cushing's disease-induced remission of severe rheumatoid arthritis. Arthritis Rheum. 37, 1826.

Ujiie, I., Ujiie, H., Iwata, H. and Shimizu, H. (2019): Clinical and immunological features of pemphigus relapse. Br. J. Dermatol. 180, 1498-1505.

Vaughan, D. F., Clay Hodgin, E., Hosgood, G. L. and Bernstein, J. A. (2010): Clinical and histopathological features of pemphigus foliaceus with and without eosinophilic infiltrates: a retrospective evaluation of 40 dogs. Vet. Dermatol. 21, 166-174.

White, S. D., Carlotti, D. N. and Pin, D. (2002): Putative drug-related pemphigus foliaceus in four dogs. Vet. Dermatol. 13, 195-202.

Zur, G. and White, S. D. (2011): Hyperadrenocorticism in 10 dogs with skin lesions as the only presenting clinical signs. J. Am. Anim. Hosp. Assoc. 47, 419-427. 